\title{
Efficacy of IL- 6 antagonists in the treatment of COVID-19 patients: further randomized controlled trials are warranted
}

\author{
Aditya K. Panda ${ }^{1}$ - Sunali Padhi ${ }^{1} \cdot$ Abhijit Pati $^{1} \cdot$ Tapan Kumar Singh Nayak $^{1}$
}

Received: 18 August 2021 / Accepted: 13 September 2021 / Published online: 15 September 2021

(c) Japan Human Cell Society 2021

Coronavirus disease-19 (COVID-19) is caused by severe acute respiratory syndrome coronavirus 2 (SARS-CoV-2), which was first reported in December 2019 in Wuhan, China. In 222 nations, approximately 209 million people are infected with SARS-CoV-2, resulting in 4.3 million fatalities. While numerous treatment strategies are being tested to treat COVID-19 individuals, none of them appears to be effective. Various monoclonal antibodies that are commonly used to treat other diseases are being evaluated for their efficacy in the treatment of COVID-19. Multiple randomized controlled studies (RCTs) have established the efficacy of IL-6 receptor antagonists, such as tocilizumab and sarilumab, in the treatment of COVID-19. A recent metaanalysis by Shankar-Hari et al. [1] using data of all published or ongoing RCTs on the efficacy of interleukin-6 receptor antagonists (tocilizumab, sarilumab, and siltuximab) investigated a possible beneficial role against all-cause mortality and secondary infections in COVID-19 patients. A total of 29 trials comprising 11,023 COVID-19 patients [tocilizumab $(\mathrm{RCTs}=19, n=8048)$ : intervention $=4299$, placebo or standard of care $=3749$; sarilumab $(\mathrm{RCTs}=9, n=2826)$ : intervention $=2073$, placebo or standard of care $=753$; siltuximab $(\mathrm{RCT}=1, n=149)$ : intervention $=77$, placebo or standard of care $=72$ ] demonstrated a substantial role for IL-6 antagonists in preventing all caused death. However, in subgroup analysis, sarilumab failed to demonstrate such a role. Additionally, the meta-analysis results were also unconvincing regarding the administration of IL-6 blockers to prevent subsequent infections.

Trial sequential analysis (TSA) is an essential statistical test to calculate the required information size of clinical trials in a meta-analysis. TSA is more advantageous over traditional meta-analysis since it reduces both type-I and type-II

Aditya K. Panda

adityarmrc@gmail.com; akpanda@khallikoteuniversity.ac.in

1 Department of Bioscience and Bioinformatics, Khallikote University, Berhampur, Odisha 761008, India errors. When the cumulative $\mathrm{Z}$ curve in the TSA reaches the needed information size, or the monitoring or futility boundary, it indicates that sufficient clinical trials have been conducted to obtain a meaningful result, and more trials are unlikely to change the observations [2-5].

The prevalence of overall mortality and secondary infections in the COVID-19 patients with or without IL-6 receptor blockers were extracted from the published article [1]. We performed the TSA to investigate the efficacy of IL-6 antagonists on overall mortality and secondary infections. The maximum type-I errors of $1 \%$ and type-II errors of $10 \%$ were fixed to calculate the required information size. The TSA for the significance of IL-6 blockers against all-cause death showed a crossing of the $\mathrm{Z}$ curve across the monitoring boundary (Fig. 1A), indicating that enough clinical trials had been performed to conclude a protective effect against COVID-19 mortality. On the other hand, subgroup analysis found that the $\mathrm{Z}$ curve for tocilizumab exceeded the required information size (Fig. 1C), whereas clinical investigations with 10,820 more participants are required to reach a valid conclusion for sarilumab (Fig. 1E).

The $\mathrm{Z}$ curve approached the futility boundary demonstrating that IL-6 blockers play no significant role in secondary infections (Fig. 1B). The subgroup analysis also found the inclusion of enough clinical trials as the cumulative $\mathrm{Z}$ curve crossed the futility line and revealed no significant role for sarilumab (Fig. 1F). However, additional clinical studies, including 14,422 COVID-19 patients, are necessary to conclude the role of tocilizumab in controlling the secondary infections because the cumulative $\mathrm{Z}$ curve did not breach the monitoring or futility boundary or reach the required level information line (Fig. 1D).

To summarize, future clinical trials with sarilumab must be done to investigate its protective effect against all causes of death, and the effectiveness of tocilizumab for minimizing secondary infections, if any, must be explored. 

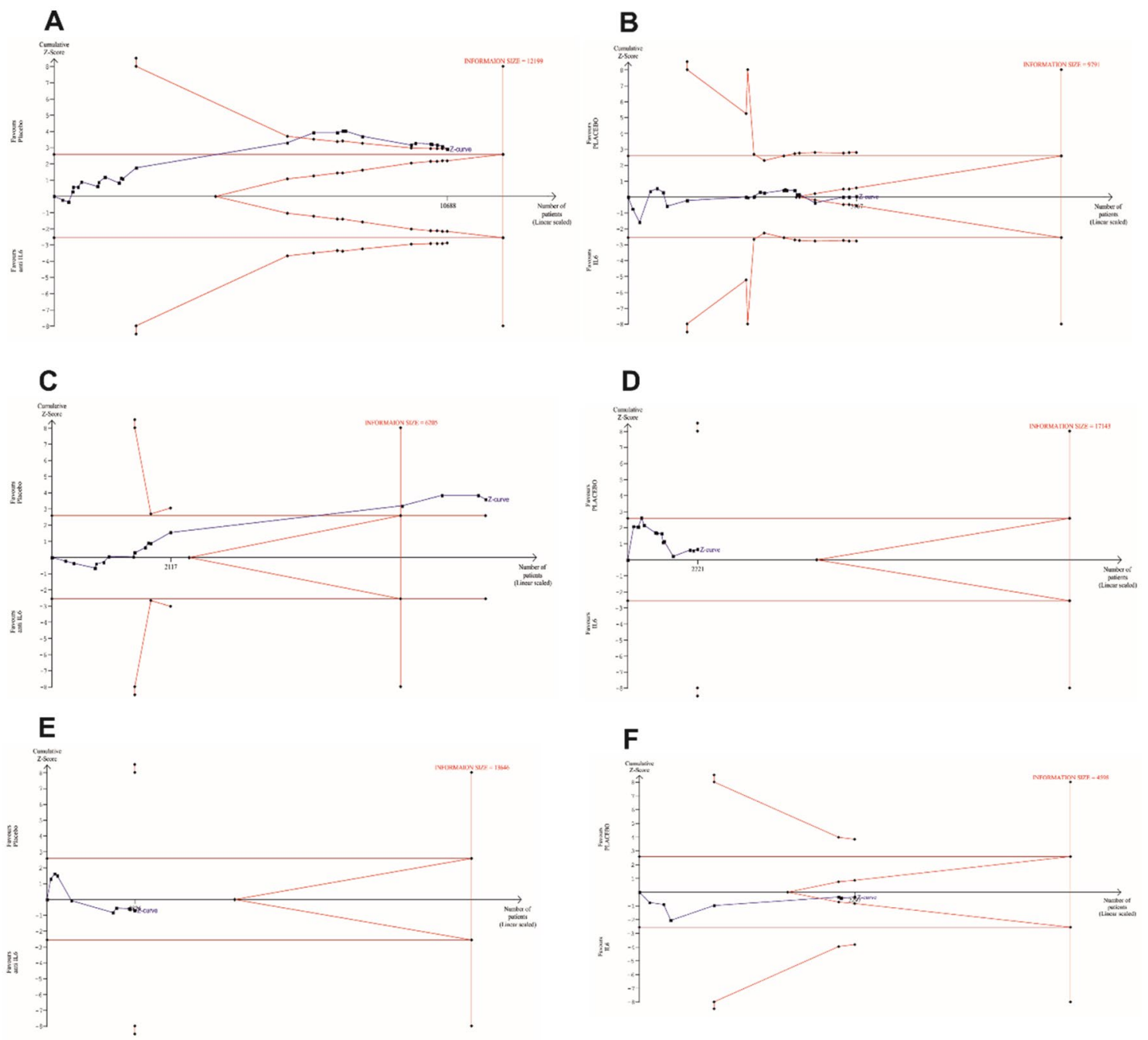

Fig. 1 Trial sequential analysis plots of the primary outcome (death) and secondary infections after SARS-CoV-2-infected subjects treated with IL-6 blockers. TSA software was employed for the analysis, and results are shown in graphs., IL-6 blockers in death (A) and secondary infection $(\mathbf{B})$, the role of Tocilizumab in death $(\mathbf{C})$ and second-

\section{Declarations}

Conflict of interest None reported.

\section{References}

1. Shankar-Hari M, Vale CL, Godolphin PJ, et al. Association between administration of IL-6 antagonists and mortality among patients hospitalized for COVID-19: a meta-analysis. JAMA. 2021. https://doi.org/10.1001/jama.2021.11330.

2. Chan JSK, Harky A. Trial sequential analysis in meta-analyses: a clinically oriented approach with real-world example. J Thorac Cardiovasc Surg. 2020. https://doi.org/10.1016/j.jtcvs.2020.06. 063.

ary infections (D), the role of sarilumab in death (E), and secondary infection $(\mathbf{F})$. To the left, the red, inward-sloping lines are the trial sequential monitoring boundaries. To the right, the red outward sloping lines are the futility region. The solid blue line is the cumulative $\mathrm{Z}$ curve

3. Thorlund K, Engstrøm J, Wetterslev J, Brok J, Imberger G, Gluud C. Trial sequential analysis (TSA). 2021. https://ctu.dk/wp-conte nt/uploads/2021/03/2017-10-10-TSA-Manual-ENG_ER.pdf.

4. Wetterslev J, Jakobsen JC, Gluud C. Trial sequential analysis in systematic reviews with meta-analysis. BMC Med Res Methodol. 2017;17:1-18. https://doi.org/10.1186/s12874-017-0315-7.

5. Padhi S, Pati A, Panda AK. Effect of ivermectin in the treatment of coronavirus disease 2019: a trial sequential analysis highlights the requirement of additional randomized, controlled trials, controlled trials. Clin Infect Dis. 2019. https://doi.org/10.1093/cid/ciab692.

Publisher's Note Springer Nature remains neutral with regard to jurisdictional claims in published maps and institutional affiliations. 